\title{
Predictive Control Strategies Operating at Fixed Switching Frequency for Input Filter Resonance Mitigation in an Indirect Matrix Converter
}

\author{
M. Rivera, S. Toledo, L. Tarisciotti, P. Wheeler and H. Dan
}

\begin{abstract}
The classic model predictive control leads to a variable switching frequency which could produce resonances in the input filter of the matrix converter, affecting the performance of the system. This paper proposes two methods to mitigate the resonance of the input filter in order to solve this issue. The first method consists in a hybrid combination of model predictive current control with instantaneous reactive power minimization and an active damping method which consists in emulating a virtual resistor in parallel to the input filter's capacitor. The second method imposes a sinusoidal source current instance to minimize the instantaneous reactive power at the input side. Both methods can be further enhanced with fixed switching frequency operations. Simulated results confirm the feasibility of the proposal demonstrating that the performance of the system is improved with source and load currents showing a significant reduction in the harmonic distortion produced by the filter resonance.
\end{abstract}

Keywords - active damping, current control, matrix converters, predictive control, finite control set model predictive control, fictitious $d c$-link.

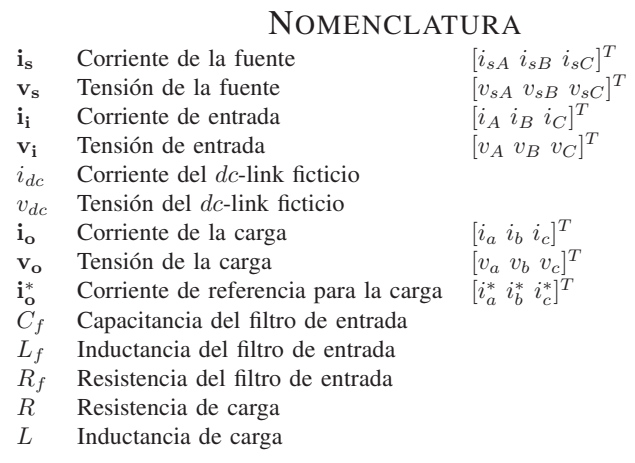

\section{INTRODUCCIÓN}

El convertidor matricial indirecto (IMC) presenta importantes ventajas como corrientes sinusoidales tanto de entrada como de salida, flujo de potencia bidireccional y desplazamiento del factor de potencia de entrada ajustable [1], [2] así como tamaño reducido y tiempo de vida elevados [3]. Por otra parte, el IMC ofrece la posibilidad de operación con una estrategia de corriente de conmutación cero en el enlace de corriente continua ( $d c$-link) el cual es más simple que las técnicas utilizadas en la topología matricial directa (DMC) [4], [5].

M. Rivera and S. Toledo are with the Department of Electromechanics and Energy Conversion, University of Talca, Curico, CHILE

H. Dan is with the School of Information Science and Engineering, Central South University, ChangSha, CHINA

L. Tarisciotti and P. Wheeler are with the Dep. of E\&E Engineering of the University of Nottingham, Nottingham, UK
El control predictivo basado en modelo (MPC) ha emergido como alternativa prometedora para el control de convertidores electrónicos de potencia [6], [7]. Esta estrategia de control utiliza el modelo matemático del sistema para predecir, para cada estado de conmutación válido, la operación de las variables a ser controladas en cada instante de muestreo. Estas predicciones son comparadas con cierta referencia mediante la evaluación de una función de costo y el estado de conmutación que genera el mínimo error entre la predicción y la referencia dada es el que se selecciona para ser aplicado en el siguiente instante de muestreo lo cual hace innecesaria además la utilización de un modulador [8]. La mayoría de las contribuciones recientes en términos del control predictivo para los IMCs han sido referentes a las diferentes topologías tales como IMC de cuatro piernas, de tres niveles y otras variantes de la mencionada topología [9], [10].

A pesar de los muchos desarrollos recientes en MPC para los convertidores de potencia, existen aún algunos tópicos los cuales son considerados como nuevos temas para la investigación. Uno de estos es el inconveniente de la frecuencia de conmutación variable que presenta la estrategia. En el MPC clásico solo un vector es elegido en cada instante de muestreo y las variables controladas presentan un alto nivel de rizado debido a la operación a frecuencia de conmutación variable, especialmente cuando la frecuencia de conmutación media se encuentra cercana a la frecuencia de resonancia del filtro de entrada. Este fenómeno afecta directamente al rendimiento del sistema tanto en la salida como en la entrada. Sin embargo, en años recientes este inconveniente se ha convertido en un tópico de investigación y algunas soluciones han sido propuestas.

En este contexto, una de las técnicas más implementadas ha sido la técnica MPC modulada (M2PC) en la cual una función de costo es utilizada para la selección de vectores óptimos adyacentes y sus correspondientes tiempos de aplicación al convertidor durante cada periodo de muestreo. La idea es emular la técnica de modulación en el espacio de vectores (SVM) utilizando MPC [11]-[13]. Haciendo esto, la estrategia de control mantiene los beneficios del MPC tradicional tales como respuesta dinámica rápida, control multiobjetivo, inclusión de restricciones y no linealidades de manera sencilla pero garantizando la operación a frecuencia de conmutación fija. Este modo de operación permite reducir el rizado de las variables controladas y mejora el desempeño general del sistema.

Esta idea ha sido también propuesta para el DMC [14][16]. Aquí, una combinación híbrida entre el MPC y SVM 
permite la operación a frecuencia de conmutación fija pero también permite el uso de todos los estados de conmutación válidos (27) del DMC, lo cual no es posible en la técnica SVM tradicional donde solo los vectores fijos son considerados [14].

A fin de resolver el inconveniente del comportamiento debido a la frecuencia de conmutación variable del MPC en el IMC el cual podría conducir a la resonancia en el filtro de entrada, este artículo presenta dos propuestas. La primera solución consiste en el control predictivo de corriente con minimización de potencia reactiva instantánea, mejorado con un método de amortiguamiento activo. La segunda solución reemplaza el término que minimiza la potencia reactiva instantánea por una función que impone una señal sinusoidal en la fuente de entrada. En ambas propuestas, la estrategia de control predictivo opera a una frecuencia de conmutación fija, permitiendo un mejor desempeño del convertidor y una forma de onda más sinusoidal en las tensiones y corrientes tanto de salida como de entrada del IMC.

\section{MOdElAdo DEl CONVERTIDOR MATRICIAL INDIRECTO}

La topología del IMC se muestra en la Figura 1. La configuración de este convertidor incluye dos etapas: el rectificador y el inversor. Esta característica constituye una interesante ventaja si se emplea un esquema de conmutación de $d c$-link cero, lo cual permite la operación segura del convertidor y un decremento de las pérdidas de conmutación. El modelo matemático del lado rectificador se define como:

$$
\begin{aligned}
& v_{d c}=\left[\begin{array}{lll}
S_{r 1}-S_{r 4} & S_{r 3}-S_{r 6} & S_{r 5}-S_{r 2}
\end{array}\right] \mathbf{v}_{\mathbf{i}} \\
& \mathbf{i}_{\mathbf{i}}=\left[\begin{array}{c}
S_{r 1}-S_{r 4} \\
S_{r 3}-S_{r 6} \\
S_{r 5}-S_{r 2}
\end{array}\right] i_{d c}
\end{aligned}
$$

Las entradas y salidas de ambas etapas son relevantes para sus estados de conmutación. Para la etapa del inversor, el modelo matemático se define como sigue:

$$
\begin{aligned}
& i_{d c}=\left[\begin{array}{lll}
S_{i 1} & S_{i 3} & S_{i 5}
\end{array}\right] \mathbf{i}_{\mathbf{o}} \\
& \mathbf{v}_{\mathbf{o}}=\left[\begin{array}{c}
S_{i 1}-S_{i 4} \\
S_{i 3}-S_{i 6} \\
S_{i 5}-S_{i 2}
\end{array}\right] v_{d c}
\end{aligned}
$$

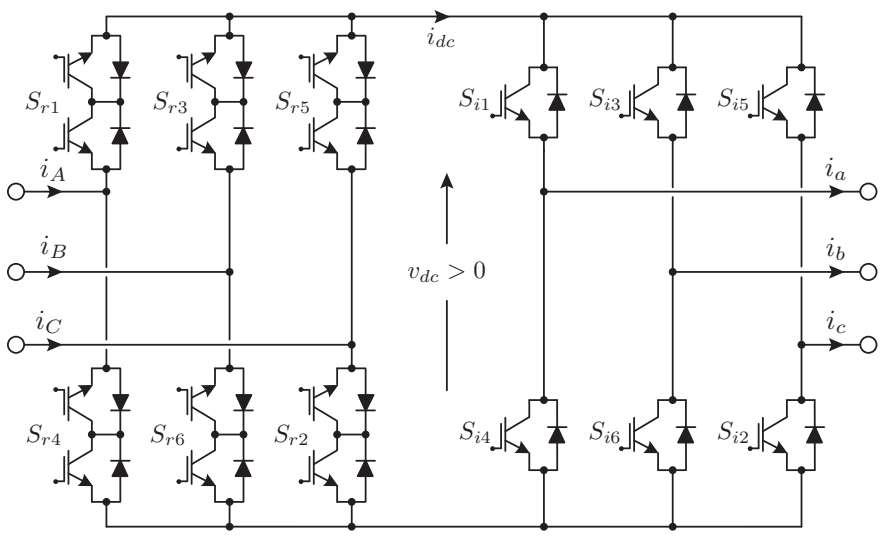

Figura 1. Circuito de potencia del convertidor matricial indirecto.
TABLA I

ESTADOS VÁLIDOS DEL LADO DEL RECTIFICADOR

\begin{tabular}{c|cccccc|ccc|c}
$\#$ & $S_{r 1}$ & $S_{r 2}$ & $S_{r 3}$ & $S_{r 4}$ & $S_{r 5}$ & $S_{r 6}$ & $i_{A}$ & $i_{B}$ & $i_{C}$ & $v_{d c}$ \\
\hline \hline 1 & 1 & 1 & 0 & 0 & 0 & 0 & $i_{d c}$ & 0 & $-i_{d c}$ & $v_{A C}$ \\
2 & 0 & 1 & 1 & 0 & 0 & 0 & 0 & $i_{d c}$ & $-i_{d c}$ & $v_{B C}$ \\
3 & 0 & 0 & 1 & 1 & 0 & 0 & $-i_{d c}$ & $i_{d c}$ & 0 & $-v_{A B}$ \\
4 & 0 & 0 & 0 & 1 & 1 & 0 & $-i_{d c}$ & 0 & $i_{d c}$ & $-v_{A C}$ \\
5 & 0 & 0 & 0 & 0 & 1 & 1 & 0 & $-i_{d c}$ & $i_{d c}$ & $-v_{B C}$ \\
6 & 1 & 0 & 0 & 0 & 0 & 1 & $i_{d c}$ & $-i_{d c}$ & 0 & $v_{A B}$
\end{tabular}

TABLA II

ESTADOS VÁLIDOS DEL LADO DEL INVERSOR

\begin{tabular}{c|cccccc|ccc|c}
$\#$ & $S_{i 1}$ & $S_{i 2}$ & $S_{i 3}$ & $S_{i 4}$ & $S_{i 5}$ & $S_{i 6}$ & $v_{u v}$ & $v_{v w}$ & $v_{w u}$ & $i_{d c}$ \\
\hline \hline 1 & 1 & 1 & 0 & 0 & 0 & 1 & $v_{d c}$ & 0 & $-v_{d c}$ & $i_{a}$ \\
2 & 1 & 1 & 1 & 0 & 0 & 0 & 0 & $v_{d c}$ & $-v_{d c}$ & $i_{a}+i_{b}$ \\
3 & 0 & 1 & 1 & 1 & 0 & 0 & $-v_{d c}$ & $v_{d c}$ & 0 & $i_{b}$ \\
4 & 0 & 0 & 1 & 1 & 1 & 0 & $-v_{d c}$ & 0 & $v_{d c}$ & $i_{b}+i_{c}$ \\
5 & 0 & 0 & 0 & 1 & 1 & 1 & 0 & $-v_{d c}$ & $v_{d c}$ & $i_{c}$ \\
6 & 1 & 0 & 0 & 0 & 1 & 1 & $v_{d c}$ & $-v_{d c}$ & 0 & $i_{a}+i_{c}$ \\
7 & 1 & 0 & 1 & 0 & 1 & 0 & 0 & 0 & 0 & 0 \\
8 & 0 & 1 & 0 & 1 & 0 & 1 & 0 & 0 & 0 & 0
\end{tabular}

Como es de esperarse, no todos los estados de conmutación posibles son válidos. Existen algunas circunscripciones que son obligatorias para la operación segura del convertidor. Las fases de entrada de la etapa del rectificar no deben cortocircuitarse, entonces, solo nueve estados son válidos para esta etapa. Las fases de salida en la etapa del inversor no deben ser desconectadas, entonces solo es posible utilizar ocho estados. Por otra parte, la tensión del $d c$-link debe ser siempre positiva a fin de permitir que los interruptores del inversor cambien. Así, los estados válidos del rectificador se reducen tan solo a tres en cada instante de muestreo, por lo cual los estados de conmutación del IMC quedan reducidos finamente a 24 estados válidos.

En la Tabla I y la Tabla II se detallan cada uno de los estados válidos tanto para el lado del rectificador como para el inversor del IMC así como su relación en términos de corrientes $\mathrm{y}$ tensiones. Con el objetivo de prevenir sobretensiones y distorsión armónica en la fuente de corriente de entrada, un filtro de entrada es necesario. Ubicando los polos del filtro en lugares determinados, es posible obtener una amplia diversidad de funciones de transferencia. En este trabajo un filtro pasa bajo de segundo orden es considerado obteniéndose las siguientes ecuaciones:

$$
\begin{gathered}
\frac{d \mathbf{i}_{\mathbf{s}}}{d t}=\frac{1}{L_{f}}\left(\mathbf{v}_{\mathbf{s}}-\mathbf{v}_{\mathbf{i}}\right)-\frac{R_{f}}{L_{f}} \mathbf{i}_{\mathbf{s}} \\
\frac{d \mathbf{v}_{\mathbf{i}}}{d t}=\frac{1}{C_{f}}\left(\mathbf{i}_{\mathbf{s}}-\mathbf{i}_{\mathbf{i}}\right)
\end{gathered}
$$

A partir de la Figura 1, es posible obtener el modelo matemático para la carga como sigue:

$$
\mathbf{v}_{\mathbf{o}}=L \frac{d \mathbf{i}_{\mathbf{o}}}{d t}+R \mathbf{i}_{\mathbf{o}}
$$

\section{Métodos de CONTROL PREDiCTIVO PROPUESTOS PARA EL IMC}

En este artículo se proponen dos esquemas de control representados en la Figura 2, los cuales permiten el control tanto del lado de entrada como el de salida del IMC [17]. 


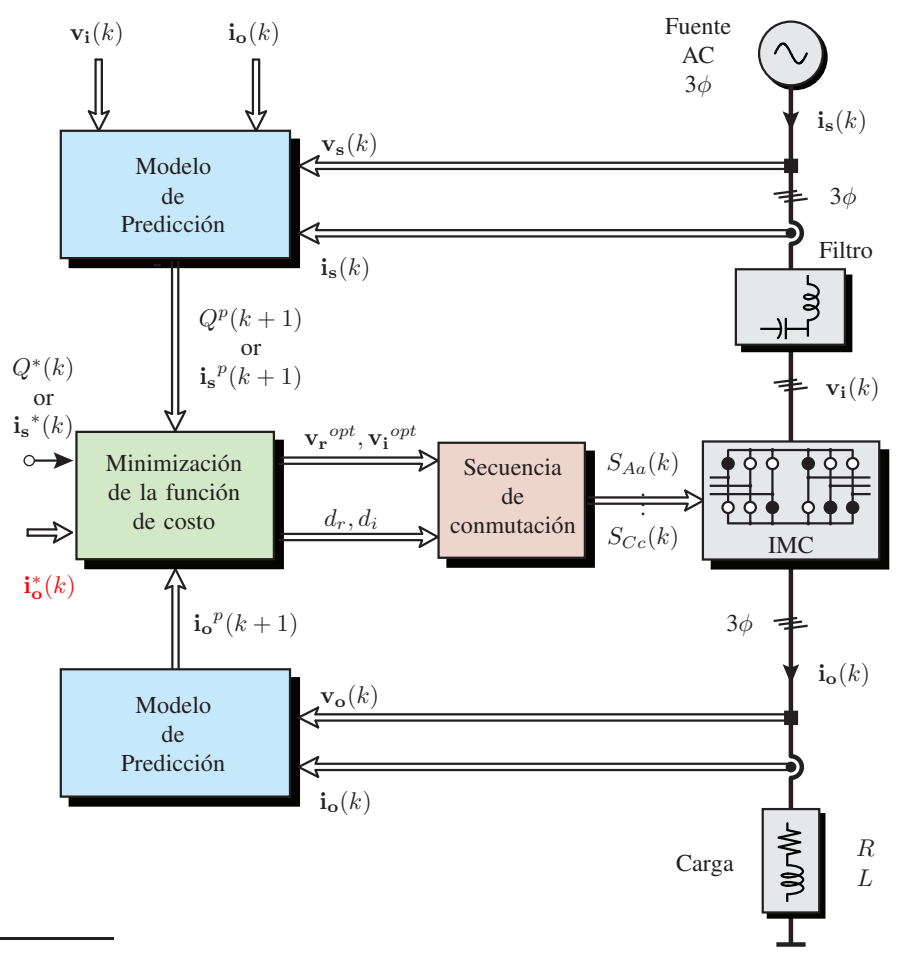

Figura 2. Control Predictivo de frecuencia de conmutación fija para el IMC

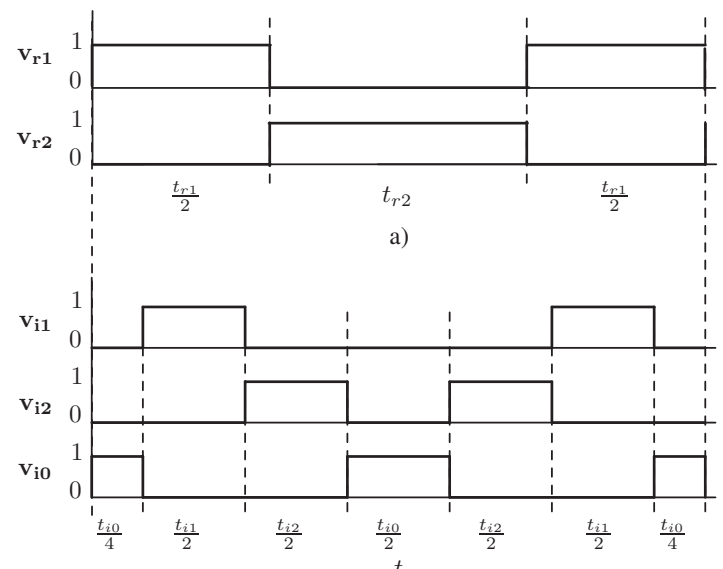

b)

Figura 3. Patrón de conmutación: a) para el lado rectificador; b) para el lado del inversor.

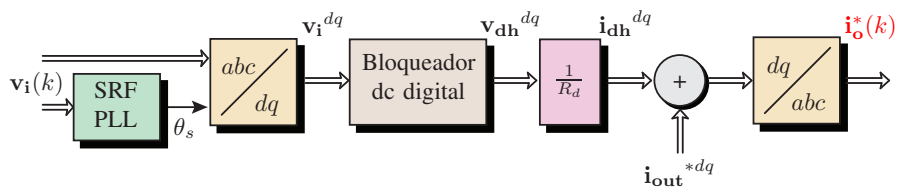

Figura 4. Implementación del amortiguamiento activo.

A cada instante de muestreo $T_{s}$, dos vectores activos son elegidos para el rectificador y dos vectores activos y un vector cero para el lado del inversor así como sus respectivos ciclos útiles los cuales son aplicados siguiendo un patrón de conmutación predefinido como se muestra en la Figura 3.

La principal contribución de este trabajo consiste en el mejoramiento de la estrategia de control predictivo de corriente operando a frecuencia de conmutación fija a una potencial frecuencia de resonancia en el filtro de entrada. Para la propuesta I, el lado de entrada es controlado imponiendo una minimización de la potencia reactiva de entrada en la función de costo y la corriente de carga es controlada imponiendo una referencia definida por el diagrama de bloques mostrado en la Figura 4 donde la implementación del amortiguamiento activo es llevado a cabo. El amortiguamiento activo no involucra mediciones o alguna otra modificación adicional en el algoritmo predictivo, y por lo tanto, es fácil de realizar. En la propuesta II, el lado de entrada del IMC es controlado imponiendo una forma de onda sinusoidal a la fuente de corriente y el lado de salida es controlado sin la implementación del método de amortiguamiento activo por lo cual, en este caso, $\mathbf{i}_{\mathbf{d h}}{ }^{d q}=0$. Considerando [9] para la predicción de tensión y corriente, es posible definir la función de costo $g_{r}$ para la propuesta I, asociada a la minimización de potencia reactiva instantánea como:

$$
g_{r}=\left[v_{s \alpha}(k+1) i_{s \beta}(k+1)-v_{s \beta}(k+1) i_{s \alpha}(k+1)\right]^{2}
$$

Para la propuesta II, la función de costo $g_{r}$ se asociada al control de la corriente de la fuente de entrada en el plano $\alpha-\beta$ el cual es definido como sigue:

$$
g_{r}=\left[i_{s \alpha}^{*}-i_{s \alpha}(k+1)\right]^{2}+\left[i_{s \beta}^{*}-i_{s \beta}(k+1)\right]^{2}
$$

Aquí, es esencial definir la corriente de referencia para la fuente del IMC, la cual resulta de definir la relación de potencia aparente en el lado de la entrada como:

$$
S_{\text {in }}=V_{i} \cdot I_{i}
$$

siendo $I_{i}$ y $V_{i}$ los valores instantáneos de la corriente y la tensión de entrada, respectivamente. La componente real de la ecuación (10) relativa a la potencia activa de entrada se muestra como función de los parámetros del lado de entrada según la ecuación siguiente:

$$
P_{i}=R e\left\{S_{i n}\right\}=3 I_{s}\left(1-8 \pi^{2} f_{s}^{2} C_{f} L_{f}\right)\left(V_{s}-R_{f} I_{s}\right)
$$

siendo $V_{s}$ la amplitud de la tensión de la fuente e $I_{s}$ la amplitud de la fundamental de la corriente de la fuente, consistente en la variable a ser calculada.

En el lado de salida, la potencia activa $P_{o}$ se obtiene como:

$$
P_{o}=3 R I_{o}^{* 2}
$$

siendo $I_{o}^{*}$ la amplitud de la corriente de referencia de salida.

La relación entre la eficiencia del convertidor $\eta$, y la potencia activa de entrada y salida se define por la ecuación:

$$
P_{i} \eta=P_{o}
$$

la cual es posible de formular en términos de las variables de entrada y salida del convertidor como sigue:

$$
I_{s}\left(1-8 \pi^{2} f_{s}^{2} C_{f} L_{f}\right)\left(V_{s}-R_{f} I_{s}\right) \eta=R I_{o}^{* 2}
$$

La ecuación (14) puede ser representada como:

$$
\left(\lambda V_{s} I_{s}-\lambda R_{f} I_{s}^{2}\right) \eta=R I_{o}^{* 2}
$$


considerando $\lambda=1-8 \pi^{2} f_{s}^{2} C_{f} L_{f}$. De la ecuación (15), es posible encontrar una relación cuadrática como la mostrada a continuación:

$$
-\lambda R_{f} I_{s}^{2}+\lambda V_{s} I_{s}-\frac{R I_{o}^{* 2}}{\eta}=0
$$

de esta manera, de la ecuación (16), es posible definir la amplitud de la corriente de la fuente como:

$$
I_{s}=\frac{-\lambda V_{s} \pm \sqrt{\left(\lambda V_{s}\right)^{2}-4 \lambda R_{f} R I_{o}^{* 2} / \eta}}{-2 \lambda R_{f}}
$$

La amplitud de referencia para la fuente de corriente es determinada en función de la eficiencia, los parámetros del filtro de entrada, la fundamental de la fuente de tensión y la amplitud de la corriente de referencia de salida. Además, es esencial aplicar un lazo de amarre de fase (PLL) para determinar la fase de la fundamental de la tensión de la fuente con el propósito de generar la referencia sinusoidal. Finalmente, la corriente de referencia resultante para la fuente se especifica como:

$$
\begin{aligned}
& i_{s A}^{*}=I_{s} \sin \left(w_{s} t+\theta\right) \\
& i_{s B}^{*}=I_{s} \sin \left(w_{s} t-2 \pi / 3+\theta\right) \\
& i_{s C}^{*}=I_{s} \sin \left(w_{s} t+2 \pi / 3+\theta\right)
\end{aligned}
$$

donde $\theta$ es el parámetro que permite un factor de potencia variable y es considerado equivalente a cero con el propósito de asegurar desplazamiento unitario del factor de potencia. Como se muestra en la Figura 5 (izquierda), es posible identificar seis sectores los cuales son definidos por dos vectores de corriente activos adyacentes $\mathbf{i}_{\mathbf{i}}$ (por ejemplo, el sector I se define por el vector $\mathbf{i}_{1}$ y el vector $\mathbf{i}_{2}$, el sector II es definido por $\mathbf{i}_{2}$ y $\mathbf{i}_{3}$, y de la misma manera para los demás). Durante cada periodo de muestreo $T_{s}$, cada par de vectores de corriente son evaluados para la función de costo $g_{r}$ lo cual significa que para cada sector, dos funciones de costo son definidas, la primera asociada a un vector de corriente $g_{r 1}$ y la otra correspondiente al vector de corriente adyacente $g_{r 2}$. Luego, estas funciones de costo son utilizadas para determinar los ciclos de trabajo que son calculados con la presunción de que los mismos son proporcionales a la inversa del valor de la función de costo relacionada, donde $K_{r}$ es una constante que debe ser especificada como sigue:

$$
\begin{aligned}
& d_{r 1}=K_{r} / g_{r 1} \\
& d_{r 2}=K_{r} / g_{r 2} \\
& d_{r 1}+d_{r 2}=1
\end{aligned}
$$

Con estos ciclos de trabajo y los valores de las funciones de costo es posible definir una nueva función de costo tal como se muestra a continuación:

$$
g_{r e c}=d_{r 1} g_{r 1}+d_{r 2} g_{r 2}
$$

Esto es llevado a cabo en cada instante de muestreo para cada uno de los seis sectores y en la etapa final, el par de vectores que minimiza la función de costo $g_{r e c}$ son elegidos como los valores óptimos $\mathbf{v}_{\mathbf{r}}{ }^{o p t}$ a ser aplicados al convertidor en el siguiente instante de muestreo. El tiempo de aplicación para cada vector se obtiene de la siguiente manera:

$$
\begin{aligned}
& t_{r 1}=d_{r 1} T_{s} \\
& t_{r 2}=d_{r 2} T_{s}
\end{aligned}
$$
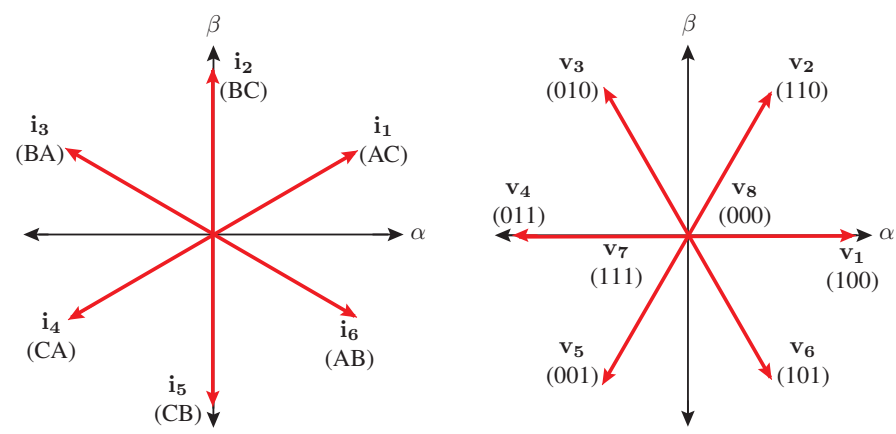

Figura 5. Espacio de vectores para la corriente y la tensión del IMC. Izquierda: espacio de vectores de corriente en el lado del rectificador, derecha: espacio de vectores de tensión para el lado del inversor.

A partir de las definiciones mostradas en las ecuaciones (4) y (7), es posible especificar el modelo de predicción del lado de salida utilizando la aproximación de Euler hacia adelante en la ecuación (7), como:

$$
\mathbf{i}_{\mathbf{o}}(k+1)=c_{1} \mathbf{v}_{\mathbf{o}}(k)+c_{2} \mathbf{i}_{\mathbf{o}}(k)
$$

donde, $c_{1}=T_{s} / L$ y $c_{2}=1-R T_{s} / L$, son constantes dependientes de los parámetros de la carga y del tiempo de muestreo $T_{s}$.

Seguidamente, la función de costo correspondiente a la etapa de salida del convertidor $g_{i}$ en el plano $\alpha-\beta$ se definde como:

$$
g_{i}=\left[i_{\alpha}^{*}-i_{\alpha}(k+1)\right]^{2}+\left[i_{\beta}^{*}-i_{\beta}(k+1)\right]^{2}
$$

Como se aprecia en la Figura 5 (derecha), es posible identificar seis sectores definidos cada uno por dos vectores de tensión activos. A cada instante de muestreo $T_{s}$, cada par de vectores de tensión y un vector cero son evaluados para la función de costo $g_{i}$ lo que significa que para cada sector se obtienen tres funciones de costo $g_{i 0}, g_{i 1}$ y $g_{i 2}$. Luego de esto, estas funciones de costo son utilizadas para determinar los ciclos de trabajo los cuales se calculan suponiendo que los mismos son proporcionales a la inversa del valor de la función de costo asociada, donde $K_{i}$ es una constante a ser determinada:

$$
\begin{gathered}
d_{i 0}=K_{i} / g_{i 0} \\
d_{i 1}=K_{i} / g_{i 1} \\
d_{i 2}=K_{i} / g_{i 2} \\
d_{i 0}+d_{i 1}+d_{i 2}=1
\end{gathered}
$$

Con los valores de la función de costo y los ciclos de trabajo calculados, se define una nueva función de costo como sigue:

$$
g_{i n v}=d_{i 1} g_{i 1}+d_{i 2} g_{i 2}
$$

De manera similar al caso anterior, esto se realiza a cada instante de muestreo para cada uno de los seis sectores y al final, el par de vectores que minimizan la función de costo $g_{i n v}$ son seleccionados como los valores óptimos $\mathbf{v}_{\mathbf{i}}{ }^{\text {opt }}$ a ser aplicados en el siguiente instante de muestreo. El tiempo de aplicación de cada uno de los vectores se obtiene como se muestra a continuación:

$$
\begin{aligned}
& t_{i 0}=d_{i 0} T_{s} \\
& t_{i 1}=d_{i 1} T_{s} \\
& t_{i 2}=d_{i 2} T_{s}
\end{aligned}
$$


(a)

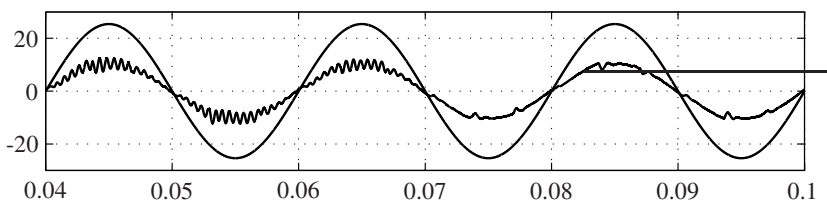

(b)
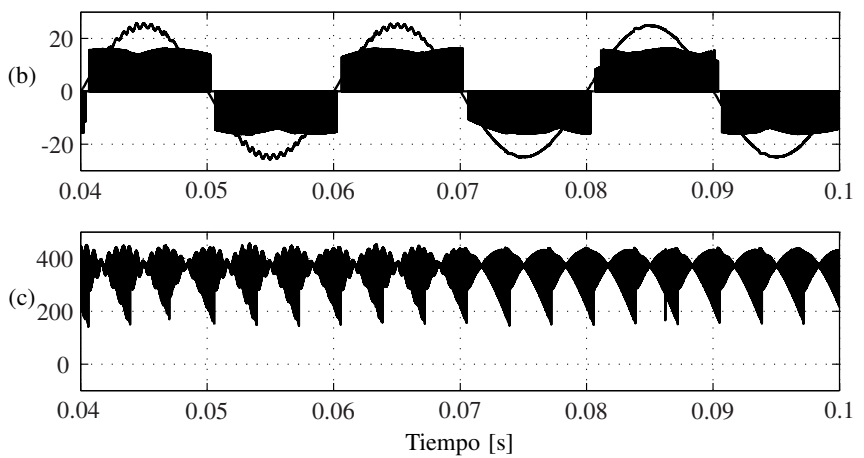

Figura 6. Resultados de simulación para la propuesta I: antes de $t=0,07$ [s] sin amortiguamiento activo, después de $t=0,07$ [s] con amortiguamiento activo: (a) tensión de la fuente $v_{s} A[\mathrm{~V} / 10]$ y corriente de la fuente $i_{s A}$ [A]; (b) tensión sobre el capacitor $v_{A}[\mathrm{~V} / 10]$ y corriente de entrada $i_{A}[\mathrm{~A}]$; c) Tensión en el $d c$-link $v_{d c}$ [V].

(a)

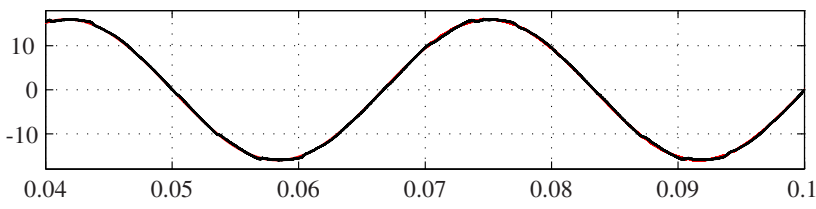

(b)
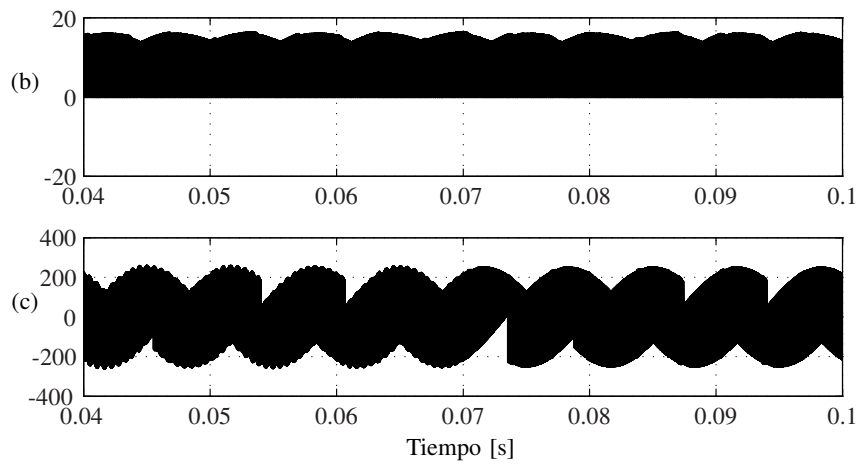

Figura 7. Resultados de simulación para la propuesta I: antes de $t=0,07$ [s] sin amortiguamiento activo, después de $t=0,07$ [s] con amortiguamiento activo: (a) corriente de carga $i_{a}[\mathrm{~A}]$; (b) corriente del $d c$-link $i_{d c}[\mathrm{~A}]$; c) Tensión de carga $v_{a}[\mathrm{~V}]$.

Después de obtener los ciclos de trabajo y seleccionar los vectores óptimos a ser aplicados en ambas etapas del convertidor (i.e. rectificador e inversor), se adopta un patrón de conmutación con el objetivo de aplicar los vectores óptimos [18] (Figura 3).

\section{Resultados}

A fin de validar la eficiencia de los métodos propuestos, se ha utilizado la herramienta Matlab-Simulink para llevar a cabo una serie de simulaciones. Ambas propuestas fueron evaluadas bajo los mismos parámetros los cuales fueron: $C_{f}=21[\mu \mathrm{F}]$, $L_{f}=400[\mu \mathrm{H}], R_{f}=0.5[\Omega], R=10[\Omega], L=10[\mu \mathrm{H}], T_{s}=50[\mu \mathrm{s}]$ a un paso de simulación de $1[\mu \mathrm{s}]$. (a)

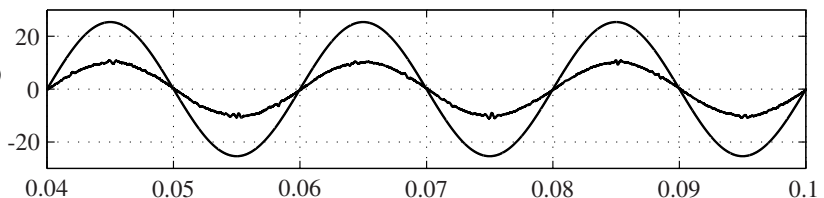

(b)
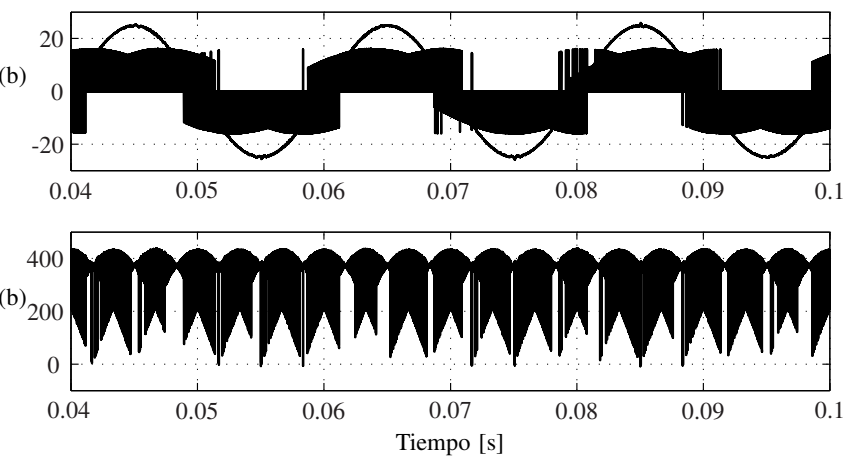

Figura 8. Resultados de simulación para la propuesta II: sin implementación de amortiguamiento activo: (a) tensión de la fuente $v_{s A}[\mathrm{~V} / 10]$ y corriente de la fuente $i_{s A}[\mathrm{~A}]$; (b) tensión en el capacitor $v_{A}[\mathrm{~V} / 10]$ y corriente de entrada $i_{A}[\mathrm{~A}]$; c) tensión en el $d c$-link $v_{d c}$ [V].

(a)

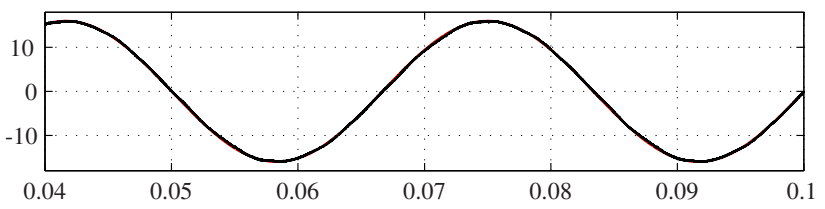

(b)
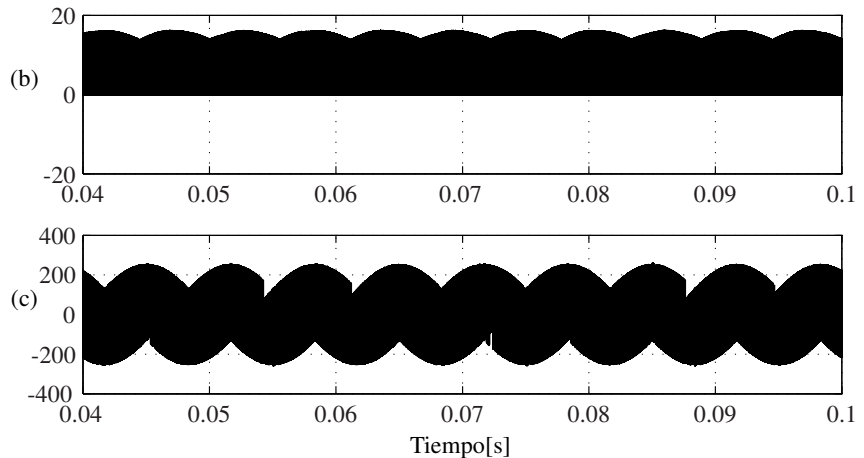

Figura 9. Resultados de simulación para la propuesta II: sin implementación de amortiguamiento activo: (a) corriente de carga $i_{a}[\mathrm{~A}]$; (b) corriente del $d c$-link $i_{d c}[\mathrm{~A}] ;$ c) tensión de carga $v_{a}[\mathrm{~V}]$.

En las figuras 6 y 7 se muestran los resultados de simulación para la propuesta I. Antes de $t=0,07$ [s] el control trabaja sin la implementación del amortiguamiento activo. En la Figura 6(a) se observa una de las corrientes de la fuente $i_{s A}$ en fase con su respectiva tensión $v_{s A}$ con un THD de $15.84 \%$. En este caso, la corriente es afectada por la resonancia del filtro de entrada lo cual es evidente en las oscilaciones que presenta esta señal.

El efecto y el desempeño de la implementación del amortiguamiento activo se refleja también en esta figura donde la presencia de armónicos de alto orden observados en la Figura 6(b) han sido eliminadas como se esperaba. En esta figura, es posible apreciar la corriente de entrada conmutada $i_{A}$, la cual es dada como función de los interruptores del rectificador y la corriente del $d c$-link $i_{d c}$. 
La Figura 6(b) muestra además el efecto de la resonancia del filtro de entrada sobre la tensión del capacitor $v_{A}$ que también presenta algunas oscilaciones debido a este fenómeno. En la Figura 6(c) se muestra la tensión del $d c$-link $v_{d c}$. La misma es función de los interruptores del rectificador y las tensiones del capacitor $\mathbf{v}_{\mathbf{i}}$. Un seguimiento altamente satisfactorio de la corriente de carga $i_{a}$ a la corriente de referencia $i_{a}^{*}$ es observado en la Figura 7(a) con una forma de onda sinusoidal y THD de $1.17 \%$. En este caso la referencia fue establecida como $I_{o}^{*}=16[\mathrm{~A}] @ 30 \mathrm{~Hz}$.

En la Figura 7(b) la corriente del $d c$-link $i_{d c}$ es también observada la cual es función del estado de los interruptores del inversor y las corrientes de carga $\mathbf{i}_{\mathbf{o}}$. La Figura 7(c) muestra la tensión en la carga que está dada en función del estado de los interruptores del inversor y la tensión del $d c$-link $v_{d c}$. Luego de $t=0,07$ [s], se aplica el amortiguamiento activo donde es posible apreciar la mejora en $i_{s A}$ que presenta una forma casi sinusoidal con THD de $6.00 \%$. En este caso el método de amortiguamiento activo reduce la distorsión armónica en la fuente en casi $65 \%$. Este efecto se refleja además en la tensión del capacitor $v_{A}$ y la tensión del $d c$-link $v_{d c}$ con una forma de onda mejorada. En el lado de la carga, la corriente de salida $i_{a}$ no se ve afectada pues la THD permanece alrededor del $1.20 \%$.

Así también, es posible de apreciar el efecto de mejora con la aplicación del amortiguamiento activo en la tensión de la carga $v_{a}$ donde casi no se observan oscilaciones.

Las figuras 8 y 9 muestran los resultados de simulación para la propuesta II. En este caso un desempeño altamente satisfactorio es observado en las variables controladas debido al efecto del modo de operación a frecuencia de conmutación fija. La corriente de la fuente $i_{s A}$ presenta una forma de onda sinusoidal con THD alrededor de $2.80 \%$. Con la propuesta II es posible obtener un desempeño superior en la corriente de la fuente en comparación con la propuesta I, pues se obtiene una valor de THD menor. Lo anterior también se refleja en el lado de la carga, donde la corriente de salida $i_{a}$ presenta un valor de THD alrededor de $0.77 \%$ con una forma de onda casi sinusoidal y un seguimiento altamente satisfactorio de la referencia $i_{a}^{*}$. En la propuesta II la implementación del amortiguamiento activo no fue necesaria dado que la corriente de la fuente es directamente controlada y forzada a seguir una referencia sinusoidal.

\section{CONCLUSión}

En este artículo han sido presentadas dos estrategias de control predictivo que mitigan los efectos de la resonancia del filtro de entrada para IMCs. Ambas estrategias operando a una frecuencia de conmutación fija a fin de mejorar el desempeño del sistema. Basado en los resultados, el método en el cual la corriente de entrada es impuesta presenta mejor desempeño que el método donde se aplica la minimización de potencia reactiva con amortiguamiento activo. Ambos métodos no requieren la implementación de sensores adicionales. Utilizando estos métodos es posible solucionar algunos inconvenientes del control predictivo tales como la operación a frecuencia de conmutación variable y sus efectos debido a la resonancia del filtro de entrada, por lo cual se mejora la forma de onda de las tensiones y las corrientes tanto en la etapa de entrada como en la de salida del convertidor. Teniendo en cuenta la implementación práctica y el modo de operación seguro del convertidor, el método de conmutación a corriente cero en el $d c$-link es utilizado para minimizar las pérdidas por conmutación en la etapa del rectificador.

\section{AgRADECIMIENTOS}

Los autores quisieran agradecer el apoyo financiero del Proyecto FONDECYT Regular 1160690 y al Proyecto MEC 80150056.

\section{REFERENCIAS}

[1] L. Empringham, J. Kolar, J. Rodriguez, P. Wheeler, and J. Clare, "Technological issues and industrial application of matrix converters: A review," Industrial Electronics, IEEE Transactions on, vol. 60, no. 10, pp. 4260-4271, Oct 2013.

[2] J. Rodriguez, M. Rivera, J. Kolar, and P. Wheeler, "A review of control and modulation methods for matrix converters," Industrial Electronics, IEEE Transactions on, vol. 59, no. 1, pp. 58-70, Jan 2012.

[3] L. C. Gili, L. O. Seman, and S. V. G. Oliveira, "Different switching sequence comparison applied to multimodular matrix converter using isvm," IEEE Latin America Transactions, vol. 16, no. 6, pp. 1595-1602, June 2018.

[4] J. Kolar, T. Friedli, J. Rodriguez, and P. Wheeler, "Review of threephase pwm ac-ac converter topologies," Industrial Electronics, IEEE Transactions on, vol. 58, no. 11, pp. 4988-5006, Nov 2011.

[5] J. J. Rodriguez, R. M. Caporal, E. Peralta, O. Carranza, and R. Ortega, "Optimal venturini modulation for a three-phase four-wire matrix converter," IEEE Latin America Transactions, vol. 14, no. 2, pp. 617-623, Feb 2016.

[6] S. Vazquez, J. Rodriguez, M. Rivera, L. G. Franquelo, and M. Norambuena, "Model predictive control for power converters and drives: Advances and trends," IEEE Transactions on Industrial Electronics, vol. 64, no. 2, pp. 935-947, Feb 2017.

[7] A. S. Lunardi, J. S. S. Chaves, and A. J. S. Filho, "Predictive direct torque control for a squirrel cage induction generator grid connected for wind energy applications," IEEE Latin America Transactions, vol. 14, no. 11, pp. 4454-4461, Nov 2016.

[8] A. G. d. Castro, W. C. d. A. Pereira, C. M. R. d. Oliveira, T. E. P d. Almeida, P. R. U. Guazzelli, J. R. B. d. A. Monteiro, and A. A. d. Oliveira Junior, "Finite control-set predictive power control of bldc drive for torque ripple reduction," IEEE Latin America Transactions, vol. 16, no. 4, pp. 1128-1135, April 2018.

[9] C. F. Garcia, M. E. Rivera, J. R. Rodriguez, P. W. Wheeler, and R. S. Peña, "Predictive current control with instantaneous reactive power minimization for a four-leg indirect matrix converter," IEEE Transactions on Industrial Electronics, vol. 64, no. 2, pp. 922-929, Feb 2017.

[10] H. M. Basri and S. Mekhilef, "Model predictive torque and flux control of induction motor fed by three level indirect matrix converter with unity power factor strategy," in 2016 IEEE 8th International Power Electronics and Motion Control Conference (IPEMC-ECCE Asia), May 2016, pp. 2557-2563.

[11] L. Tarisciotti, A. Formentini, A. Gaeta, M. Degano, P. Zanchetta, R. Rabbeni, and M. Pucci, "Model predictive control for shunt active filters with fixed switching frequency," IEEE Transactions on Industry Applications, vol. 53, no. 1, pp. 296-304, Jan 2017.

[12] S. A. Odhano, A. Formentini, P. Zanchetta, R. Bojoi, and A. Tenconi, "Finite control set and modulated model predictive flux and current control for induction motor drives," in IECON 2016 - 42nd Annual Conference of the IEEE Industrial Electronics Society, Oct 2016, pp. 2796-2801.

[13] S. Toledo, M. Rivera, J. Muñoz, R. Peña, J. Riveros, and R. Gregor, "Fixed switching frequency predictive control for a multi-drive indirect matrix converter system," in 2017 IEEE Southern Power Electronics Conference (SPEC), Dec 2017, pp. 1-6.

[14] H. Huisman, M. Roes, and E. Lomonova, "Continuous control set space vector modulation for the $3 \times 3$ direct matrix converter," in 2016 18th European Conference on Power Electronics and Applications (EPE'16 ECCE Europe), Sept 2016, pp. 1-10. 
[15] M. Vijayagopal, L. Empringham, L. de Lillo, L. Tarisciotti, P. Zanchetta, and P. Wheeler, "Control of a direct matrix converter induction motor drive with modulated model predictive control," in 2015 IEEE Energy Conversion Congress and Exposition (ECCE), Sept 2015, pp. 43154321.

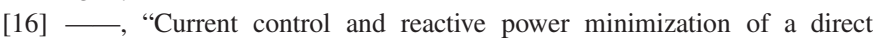
matrix converter induction motor drive with modulated model predictive control," in 2015 IEEE International Symposium on Predictive Control of Electrical Drives and Power Electronics (PRECEDE), Oct 2015, pp. 103-108.

[17] M. Rivera, H. Dan, L. Tarisciotti, and P. Wheeler, "Indirect model predictive control strategy with active damping implementation for a direct matrix converter operating at fixed switching frequency," in 2017 CHILEAN Conference on Electrical, Electronics Engineering, Information and Communication Technologies (CHILECON), Oct 2017, pp. 1-6.

[18] S. Vazquez, A. Marquez, R. Aguilera, D. Quevedo, J. Leon, and L. Franquelo, "Predictive optimal switching sequence direct power control for grid connected power converters," Industrial Electronics, IEEE Transactions on, vol. PP, no. 99, pp. 1-1, 2014.

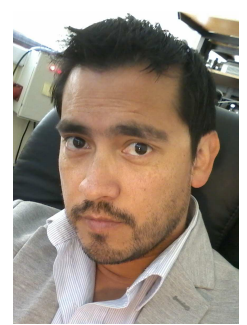

Marco Rivera (S'09-M'11) was born in Talca, Chile, in 1982. He received the B.Sc. degree in electronics engineering and the M.Sc. degree in electrical engineering from the Universidad de Concepción, Concepción, Chile, in 2007 and 2008, respectively, and the Ph.D. degree from the Department of Electronics Engineering, Universidad Técnica Federico Santa María, Valparaíso, Chile, in 2011. Since 2013 is with the Energy Conversion and Power Electronics Research Group at the Universidad de Talca. He is currently an Associate Professor with the Department of Industrial Technologies at the Universidad de Talca, Curicó, Chile. His main research areas are digital control applied to power electronics, matrix converters, predictive control and control of power converters for renewable energy applications.

Prof. Rivera was recipient of the Best $\mathrm{PhD}$ Thesis Award 2012, award given by the Chilean Academy of Science for PhD thesis developed in 2011 by national and foreign students in any Exact or Nature Sciences Program in Chile. In August 2015, Prof. Rivera was awarded with the Outstanding Engineer 2015 Award of the Electrical-Electronics Industry Association and the IEEE-Chile Section and also he received the Second Prize Paper Award in the 2015 IEEE Journal of Emerging and Selected Topics in Power Electronics.

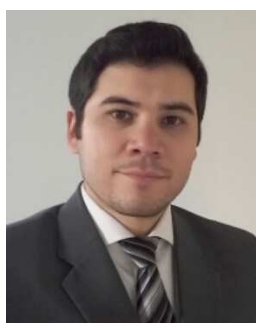

Sergio Toledo (S'09-M'12) was born in Luque, Paraguay in 1985. He received the Electronic Engineer degree from Universidad Nacional de Asunción, Paraguay in 2011 and the M.Sc. degree in Electronics and Telecomunications from Centro de Investigación Científica y Educación Superior de Ensenada, México, in 2014. His research interests include nonlinear control systems, new converter topologies and control of power converters. He is currently a Phd student in the Department of Electrical Engineering at the Universidad de Talca, Curicó, Chile.

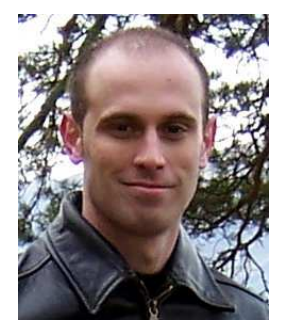

Luca Tarisciotti (S'12-M'15) received the Master's degree in electronic engineering from The University of Rome "Tor Vergataiin 2009 and his Ph.D. degree in Electrical and Electronic Engineering from the PEMC group, University of Nottingham in 2015. $\mathrm{He}$ is currently working as Research Fellow at the University of Nottingham, UK. His research interests includes matrix converters, DC/DC converters, multilevel converters, advanced modulation schemes, and advanced power converter control. international conferences and journals.

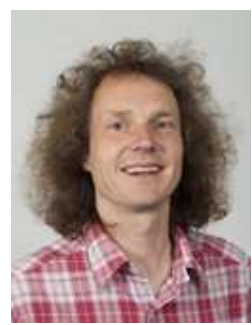

Patrick W. Wheeler received his BEng [Hons] degree in 1990 from the University of Bristol, UK. $\mathrm{He}$ received his $\mathrm{PhD}$ degree in Electrical Engineering for his work on Matrix Converters from the University of Bristol, UK in 1994. In 1993 he moved to the University of Nottingham and worked as a research assistant in the Department of Electrical and Electronic Engineering. In 1996 he became a Lecturer in the Power Electronics, Machines and Control Group at the University of Nottingham, UK. Since January 2008 he has been a Full Professor in the same research group. He is currently Head of the Department of Electrical and Electronic Engineering at the University of Nottingham. He is an IEEE PELs 'Member at Large' and an IEEE PELs Distinguished Lecturer. He has published 400 academic publications in leading international conferences and journals.

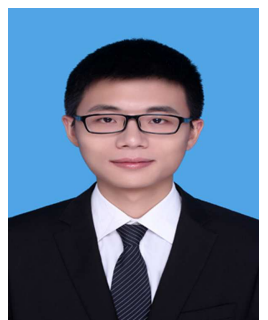

Hanbing Dan was born in Hubei, China, in 1991. He received the B.S. degree in Automation, and $\mathrm{Ph} . \mathrm{D}$ degree in Control Science and Engineering from Central South University, Changsha, China, in 2012, and 2017, respectively. He is currently an associate professor with the School of Information Science and Engineering, Central South University, China. He is a visiting researcher in Faculty of Engineering at the University of Nottingham, Unitied Kingdom between Nevember 2016 and Nevember 2017. His research interests include matrix converter, finite control set-model predictive control, fault diagnosis and fault tolerant of power converter, wireless power transfer. 